\title{
Spectra of Generalized Cesàro Operators Acting on Growth Spaces
}

\author{
Bartosz Malman(1)
}

\begin{abstract}
We study the spectrum of generalized Cesàro operators $T_{g}$ acting on the class of growth spaces $A^{-\alpha}$. We show how the problem of determining the spectrum is related to boundedness of standard weighted Bergman projections on weighted $L^{\infty}$-spaces. Using this relation we establish some general spectral properties of these operators, and explicitly compute the spectrum for a large class of symbols $g$.

Keywords. Generalized Cesàro operators, Volterra-type integral operators, Weighted L-infinity spaces, Weighted Bergman projections.
\end{abstract}

\section{Introduction and Statements of the Main Results}

Let $\mathbb{D}$ denote the open unit disk of the complex plane $\mathbb{C}$. For a fixed analytic function $g: \mathbb{D} \rightarrow \mathbb{C}$ with $g(0)=0$ we define the corresponding generalized Cesàro operator $T_{g}$ acting on an analytic function $f: \mathbb{D} \rightarrow \mathbb{C}$ by the formula

$$
T_{g} f(z)=\int_{0}^{z} g^{\prime}(\zeta) f(\zeta) d \zeta .
$$

For the particular choice of $g(z)=\log \left(\frac{1}{1-z}\right)$ we obtain the classical (shifted) Cesàro operator, which can alternatively be defined by its action on the Taylor coefficients of $f(z)=\sum_{n=0}^{\infty} a_{n} z^{n}$ by the formula

$$
\sum_{n=0}^{\infty} a_{n} z^{n} \mapsto \sum_{n=0}^{\infty} b_{n} z^{n+1},
$$

where

$$
b_{n}=\frac{1}{n+1} \sum_{k=0}^{n} a_{k} .
$$

The action of generalized Cesàro operators on classical spaces of analytic functions has been studied in a number of articles. It was noted in [14] that $T_{g}$ is bounded on the Hardy space $H^{2}$ if and only if $g$ is a function of bounded mean oscillation, and this fact was used to obtain a short proof of the analytic John-Nirenberg inequality. Further results on boundedness and compactness 
of $T_{g}$ acting on the Hardy spaces $H^{p}$ for $p \neq 2$ have been obtained in [5], and a characterization of the spectrum of $T_{g}$ acting on those spaces is available in [3]. Further properties of the spectrum of $T_{g}$ acting on $H^{2}$ are described in [19]. The corresponding questions have also been studied in the context of the Bergman spaces (see $[2,6]$ ). More recently, $T_{g}$ operators with entire symbols $g: \mathbb{C} \rightarrow \mathbb{C}$ acting on spaces of entire functions have been studied in $[8,10,11]$. A study of the spectrum of a certain normalized version of $T_{g}$ acting on a class of Banach spaces of analytic function satisfying some natural assumptions is carried out in [4]. The two excellent survey articles [1] and [16] contain more references and mention how this class of operators appear in other parts of analysis.

The purpose of this article is to study the spectrum of $T_{g}$ acting on the so-called growth spaces. For $\alpha>0$, the growth space $A^{-\alpha}$ is the Banach space of analytic functions defined in $\mathbb{D}$ for which the quantity

$$
\|f\|_{-\alpha}:=\sup _{z \in \mathbb{D}}\left(1-|z|^{2}\right)^{\alpha}|f(z)|
$$

is finite. Growth spaces appear in several contexts, perhaps most importantly in the study of zero sequences and interpolating sequences for the classical Bergman spaces (see [13, Chapter 4 and 5]). The closure of analytic polynomials in the norm $\|\cdot\|_{-\alpha}$ is denoted by $A_{0}^{-\alpha}$ and consists precisely of those functions $f \in A^{-\alpha}$ for which

$$
\lim _{r \rightarrow 1} \sup _{r<|z|<1}\left(1-|z|^{2}\right)^{\alpha}|f(z)|=0 .
$$

The space $A_{0}^{-\alpha}$ has the big advantage of being separable, and it turns out that $T_{g}$ exhibits largely the same behaviour on $A^{-\alpha}$ and $A_{0}^{-\alpha}$. As we will see in Sect. $3, T_{g}$ is simultaneously bounded or compact on both spaces, and the spectrum of $T_{g}$ is the same for both spaces. The boundedness and compactness questions have already been studied in [17], where it is shown that $T_{g}$ is bounded on $A^{-\alpha}$ if and only if $g$ satisfies

$$
\|g\|_{\mathcal{B}}:=\sup _{z \in \mathbb{D}}\left(1-|z|^{2}\right)\left|g^{\prime}(z)\right|<\infty
$$

that is, if and only if $g$ is contained in the Bloch space $\mathcal{B}$. The operator is compact if and only if $g \in \mathcal{B}_{0}$, the little Bloch space, which is the subspace of $\mathcal{B}$ consisting of functions which satisfy

$$
\lim _{r \rightarrow 1} \sup _{r<|z|<1}\left(1-|z|^{2}\right)\left|g^{\prime}(z)\right|=0 .
$$

Our study of the spectrum $\sigma\left(T_{g} \mid A^{-\alpha}\right)$ follows an idea of [2] which translates the spectral problem into an equivalent problem of characterizing weights $w: \mathbb{D} \rightarrow(0, \infty)$ with a certain property. It starts with the observation that for $\lambda \in \mathbb{C} \backslash\{0\}$ the unique analytic solution $h$ to the equation

$$
\left(1-\lambda^{-1} T_{g}\right) h=f
$$

is given by

$$
h(z)=R_{\lambda, g} f(z):=f(0) e^{\frac{g(z)}{\lambda}}+e^{\frac{g(z)}{\lambda}} \int_{0}^{z} e^{-\frac{g(\zeta)}{\lambda}} f^{\prime}(\zeta) d \zeta .
$$


It is easy to see that the operator $T_{g}-\lambda$ is injective, and hence it will be invertible on any Banach space $X$ if and only if the operator $R_{\lambda, g}$ acts boundedly on $X$. In the case $X=A^{-\alpha}$, this in turn implies an equivalence of norms on a certain Banach space of analytic functions which we will now describe. To this end, let $w$ be a weight on $\mathbb{D}$. For us, this will mean a strictly positive continuous function $w: \mathbb{D} \rightarrow(0, \infty)$. Consider the space $L_{w}^{\infty}$ consisting of (equivalence classes of) measurable functions $f$ defined in $\mathbb{D}$ which satisfy $\|w f\|_{\infty}<\infty$, where $\|\cdot\|_{\infty}$ denotes the usual essential supremum norm. Let $H_{w}^{\infty}$ be the closed subspace of $L_{w}^{\infty}$ consisting of analytic functions. For $\lambda \in \mathbb{C} \backslash\{0\}$ let

$$
w(z)=\left|e^{\frac{g(z)}{\lambda}}\right|\left(1-|z|^{2}\right)^{\alpha} .
$$

It turns out (see Proposition 5.1 below) that $\lambda$ is in the resolvent set $\rho\left(T_{g} \mid A^{-\alpha}\right.$ ) if and only if for $f \in H_{w}^{\infty}$ we have the equivalence of norms

$$
\sup _{z \in \mathbb{D}} w(z)|f(z)| \sim \sup _{z \in \mathbb{D}} w(z)\left(1-|z|^{2}\right)\left|f^{\prime}(z)\right|+|f(0)| .
$$

We are thus lead to studying weights $w$ for which such an equivalence holds. We show in Sect. 4 that the existence of such a norm equivalence is connected to the boundedness on $L_{w}^{\infty}$ of the weighted Bergman projections. For $\delta>-1$, the weighted Bergman projection $P_{\delta}$ is given by

$$
P_{\delta} f(z)=(\delta+1) \int_{\mathbb{D}} f(\zeta) \frac{\left(1-|\zeta|^{2}\right)^{\delta}}{(1-z \bar{\zeta})^{2+\delta}} d A(\zeta)
$$

where $d A$ denotes the area measure on $\mathbb{D}$. For the class of bounded and differentiable weights satisfying for some constant $k_{w}>0$ the inequality

$$
(1-|z|)|\nabla w(z)| \leq k_{w} w(z)
$$

we prove the following theorem (see Sect. 4 for the definition of $\tilde{P}_{\delta}$ appearing below).

Theorem 4.5. Let $w: \mathbb{D} \rightarrow(0, \infty)$ be a bounded and differentiable weight which satisfies (3). The following are equivalent:

(i) The operator $P_{\delta}$ is bounded on $L_{w}^{\infty}$ for some $\delta>-1$.

(ii) The operator $\tilde{P}_{\delta}$ is bounded on $L_{w}^{\infty}$ for some $\delta>-1$

(iii) There exists $\delta>-1$ such that

$$
\sup _{z \in \mathbb{D}} w(z) \int_{\mathbb{D}} \frac{1}{w(\zeta)} \frac{\left(1-|\zeta|^{2}\right)^{\delta}}{|1-z \bar{\zeta}|^{\delta+2}} d A(\zeta)<\infty .
$$

(iv) For each $n \geq 1$

$$
\sup _{z \in \mathbb{D}}\left(1-|z|^{2}\right)^{n} w(z)\left|f^{(n)}(z)\right|+\sum_{k=0}^{n-1}\left|f^{(k)}(0)\right|
$$

defines an equivalent norm on $H_{w}^{\infty}$.

The first three conditions of the above theorem are equivalent, with the same constant $\delta$, even without the assumption that (3) holds (see Theorem 4.1). The fourth condition is on the other hand crucial for our further study of the spectrum of $T_{g}$ on the growth spaces. We note that the general problem 
of boundedness of weighted Bergman projections on $L_{w}^{\infty}$ has been studied in $[9]$.

Results of Sect. 4 are applied to the spectral problem in Sect. 5. The main difficulty is a technical one of extending (2) to higher derivatives, i.e., to establish that condition (iv) of Theorem 4.5 holds for the weight given by (1) whenever $\lambda \in \rho\left(T_{g} \mid A^{-\alpha}\right)$. The main tool here is an operator interpolation theorem which we prove in Sect. 2 (see Theorem 2.2 below), and the result is the following characterization of the spectrum of $T_{g}$ acting on $A^{-\alpha}$.

Theorem 5.3. Let $g \in \mathcal{B}, \lambda \in \mathbb{C} \backslash\{0\}$ and

$$
w(z)=\left|e^{\frac{g(z)}{\lambda}}\right|\left(1-|z|^{2}\right)^{\alpha} .
$$

The following are equivalent:

(i) $\lambda \in \rho\left(T_{g} \mid A^{-\alpha}\right)$.

(ii) For some $\delta>-1$, the weight $w$ satisfies

$$
\sup _{z \in \mathbb{D}} w(z) \int_{\mathbb{D}} \frac{1}{w(\zeta)} \frac{\left(1-|\zeta|^{2}\right)^{\delta}}{|1-z \bar{\zeta}|^{\delta+2}} d A(\zeta)<\infty .
$$

This characterization allows us to explicitly compute the spectrum for a class of symbols $g \in \mathcal{B}$. It turns out, precisely as is the case for many other Banach spaces $X$, that the spectrum of $T_{g}$ acting on $X=A^{-\alpha}$ satisfies the equality

$$
\sigma\left(T_{g} \mid X\right)=\{0\} \bigcup \overline{\left\{\lambda \in \mathbb{C} \backslash\{0\}: e^{g / \lambda} \notin X\right\}}
$$

whenever $g$ is the primitive of a rational function. Moreover, we obtain the following spectral stability result.

Theorem 5.4. Let $g, h \in \mathcal{B}$ and assume that $\sigma\left(T_{h} \mid A^{-\alpha}\right)=\{0\}$. Then

$$
\sigma\left(T_{g+h} \mid A^{-\alpha}\right)=\sigma\left(T_{g} \mid A^{-\alpha}\right) .
$$

This allows us to extend (4) to the case when $g$ is the sum of a primitive of a rational function with a bounded analytic function and a function in $\mathcal{B}_{0}$ (see Theorem 5.6).

For a general Banach space $X$ and a general symbol $g$, the equality in (4) does not hold. This is shown in [2] in the case when $X$ is any of the standard Bergman spaces. By relating condition (ii) of Theorem 5.3 to the classical Békollé weight condition, we show that the example in [2] can be adapted to the case considered here, so that the spectrum of $T_{g}$ on $A^{-\alpha}$ can indeed be much larger than (4).

\section{Preliminaries}

\subsection{An Integral Kernel Estimate}

The following well-known estimate will be used frequently below.

Proposition 2.1. For any $\delta>-1$ and any $s>0$ there exists a constant $C>0$ such that for $z \in \mathbb{D}$ we have

$$
\int_{\mathbb{D}} \frac{\left(1-|\zeta|^{2}\right)^{\delta}}{|1-z \bar{\zeta}|^{\delta+2+s}} d A(\zeta) \leq \frac{C}{(1-|z|)^{s}} .
$$


For a proof, see [13, Theorem 1.7].

\subsection{Equivalent Norms}

For any $n \geq 0$, the space $A^{-\alpha}$ can equivalently be normed by

$$
\sup _{z \in \mathbb{D}}\left(1-|z|^{2}\right)^{\alpha+n}\left|f^{(n)}(z)\right|+\sum_{k=0}^{n-1}\left|f^{(k)}(0)\right|
$$

(see Theorem 5.5 in [12]). We will often use these norms without further reference.

\subsection{Duality Relations}

Let $L^{1}(\mathbb{D})$ be the Lebesgue space of (equivalence classes of ) measurable functions which are integrable with respect to $d A$. It is a consequence of the results of [15] that the dual space $\left(A_{0}^{-\alpha}\right)^{*}$ can be identified with the quotient space $L^{1}(\mathbb{D}) / N$, where

$$
N=\left\{f \in L^{1}(\mathbb{D}): \int_{\mathbb{D}} f(\zeta)\left(1-|\zeta|^{2}\right)^{\alpha} h(\zeta) d A(\zeta)=0, \forall h \in A^{-\alpha}\right\} .
$$

Moreover, it is also proved in [15] that the dual space $\left(L^{1}(\mathbb{D}) / N\right)^{*}$ can be identified with $A^{-\alpha}$. The duality pairing is in both cases given by

$$
\int_{\mathbb{D}} f(\zeta)\left(1-|\zeta|^{2}\right) h(\zeta) d A(\zeta),
$$

where $f \in L^{1}(\mathbb{D}) / N$ and $h \in A^{-\alpha}$ or $h \in A_{0}^{-\alpha}$.

It is clear from this characterization that a sequence $\left\{f_{n}\right\}_{n=1}^{\infty}$ in $A_{0}^{-\alpha}$ converges weakly to $f$ if and only if the norms $\left\|f_{n}\right\|_{-\alpha}$ stay bounded and $f_{n}(z) \rightarrow f(z)$ for each $z \in \mathbb{D}$ as $n \rightarrow \infty$, uniformly on compact subsets of $\mathbb{D}$. As the dual space of $L^{1}(\mathbb{D}) / N$, the space $A^{-\alpha}$ can be equipped with the usual weak-* topology, and similarly $\left\{f_{n}\right\}_{n=1}^{\infty}$ in $A^{-\alpha}$ converges weak-* to a function $f$ if and only if the norms $\left\|f_{n}\right\|_{-\alpha}$ stay bounded and $f_{n}(z) \rightarrow f(z)$ uniformly on compact subsets of $\mathbb{D}$. In particular, for $r \in(0,1)$ the dilations $f_{r}(z)=f(r z)$ of any function $f \in A^{-\alpha}$ converge weak-* to $f$ as $r \rightarrow 1$.

\subsection{Interpolation of Operators on Growth Spaces}

In Sect. 5 we will need the following result on interpolation of linear operators between two growth spaces. The proof consists of applying the basic ideas of the well-known complex interpolation method.

Theorem 2.2. Let $R: A_{0}^{-\beta} \rightarrow A_{0}^{-\beta}$ be a bounded linear operator. If for some $\alpha \in(0, \beta)$ the operator $R$ also maps $A_{0}^{-\alpha}$ boundedly into itself, then the operator $R$ maps $A_{0}^{-\gamma}$ boundedly into itself for any $\gamma \in(\alpha, \beta)$.

Proof. Let $C_{0}(\mathbb{D})$ denote the Banach space of functions continuous in $\overline{\mathbb{D}}$ that vanish on the boundary $\partial \mathbb{D}=\mathbb{T}$, and let

$$
S=S(\alpha, \beta)=\{z \in \mathbb{C}: \alpha<\operatorname{Re} z<\beta\} .
$$

Fix a function $f \in C_{0}(\mathbb{D})$. For $z \in \bar{S}$ let

$$
h_{z}(\lambda)=\int_{\mathbb{D}} f(\zeta) \frac{\left(1-|\zeta|^{2}\right)^{\beta-z}}{(1-\lambda \bar{\zeta})^{\beta+2}} d A(\zeta), \quad \lambda \in \mathbb{D}
$$


Proposition 2.1 implies that $h_{z} \in A_{0}^{-\operatorname{Re} z} \subseteq A_{0}^{-\beta}$ and that the norms $\left\|h_{z}\right\|_{-\beta}$ are bounded uniformly in $z \in \bar{S}$ by a constant multiple of $\|f\|_{\infty}$, i.e., there exists a constant $C>0$ such that for all $\lambda \in \mathbb{D}$ and all $z \in \bar{S}$ we have the estimate $\left|h_{z}(\lambda)\right| \leq C\|f\|_{\infty}(1-|\lambda|)^{-\beta}$. In particular, the family $\left\{h_{z}\right\}_{z \in \bar{S}}$ is bounded uniformly on compact subsets of $\mathbb{D}$. It is also clear that $h_{z_{n}}(\lambda) \rightarrow$ $h_{z}(\lambda)$ uniformly on compact subsets of $\mathbb{D}$ if $z_{n} \rightarrow z \in \bar{S}$, and hence in that case $h_{z_{n}} \rightarrow h_{z}$ weakly in $A_{0}^{-\beta}$.

For a fixed $\epsilon>0$ consider the function $G_{\epsilon}: \bar{S} \rightarrow C_{0}(\mathbb{D})$ given by

$$
G_{\epsilon}(z)=\exp \left(\frac{-\epsilon}{1-|\cdot|}\right)\left(1-|\cdot|^{2}\right)^{z}\left(R h_{z}\right)(\cdot) .
$$

Note that the exponential factor in the definition of $G_{\epsilon}$ ensures that

$$
\sup _{z \in \bar{S}}\left\|G_{\epsilon}(z)\right\|_{\infty}<\infty \text {. }
$$

If $z_{n} \rightarrow z \in \bar{S}$, then the above paragraph and the fact that $R$ preserves weak convergence of sequences implies that $R h_{z_{n}} \rightarrow R h_{z}$ weakly in $A_{0}^{-\beta}$. Then $R h_{z_{n}}(\lambda) \rightarrow R h_{z}(\lambda)$ uniformly on compact subsets of $\mathbb{D}$, and so $\| G_{\epsilon}\left(z_{n}\right)-$ $G_{\epsilon}(z) \|_{\infty} \rightarrow 0$ by the rapid decay of $\exp (-\epsilon /(1-|\lambda|))$. This shows continuity of $G_{\epsilon}$. We claim that $G_{\epsilon}$ is analytic in $S$. We will complete the proof of the theorem under this assumption, and prove analyticity of $G_{\epsilon}$ next. The assumption of boundedness of $R$ on $A_{0}^{-\alpha}$ together with Proposition 2.1 implies that for all $y \in \mathbb{R}$, we have

$$
(1-|\lambda|)^{\alpha} R h_{\alpha+i y}(\lambda) \leq C\|f\|_{\infty}
$$

and consequently

$$
\left\|G_{\epsilon}(\alpha+i y)\right\|_{\infty} \leq C\|f\|_{\infty}
$$

where $C>0$ depends only on the norm of the operator $R$ on $A_{0}^{-\alpha}$ but not on $\epsilon>0$. In the same way we obtain

$$
\left\|G_{\epsilon}(\beta+i y)\right\|_{\infty} \leq C_{1}\|f\|_{\infty},
$$

$C_{1}>0$ depending on the norm of $R$ on $A_{0}^{-\beta}$ but being independent of $\epsilon>0$. By the vector-valued generalization of the classical Hadamard's three lines theorem we get that $\left\|G_{\epsilon}(z)\right\| \leq C_{2}\|f\|_{\infty}$ for all $z \in S$, with a constant $C_{2}>0$ independent of $\epsilon>0$. Now let $g \in A_{0}^{-\gamma}, \alpha<\gamma<\beta$ and put $f(\zeta)=g(\zeta)\left(1-|\zeta|^{2}\right)^{\gamma} \in C_{0}(\mathbb{D})$. In the above notation we have that $h_{\gamma}=g$, and we conclude that

$$
\exp \left(\frac{-\epsilon}{1-|\lambda|^{2}}\right)(1-|\lambda|)^{\gamma}|R g(\lambda)| \leq\left\|G_{\epsilon}(\gamma)\right\|_{\infty} \leq C_{2}\|g\|_{-\gamma} .
$$

Letting $\epsilon$ tend to zero and taking the supremum over $\lambda \in \mathbb{D}$ shows that $R$ is bounded on $A_{0}^{-\gamma}$.

It remains to prove that $G_{\epsilon}: S \rightarrow C_{0}(\mathbb{D})$ is analytic. Recall that if $\Omega \subseteq \mathbb{C}$ and $X$ is a Banach space, then to verify that a function $F: \Omega \rightarrow X$ is analytic it suffices to verify that $z \mapsto \phi(F(z))$ is scalar-valued analytic for any $\phi \in X^{*}$ (see [18, p. 266, Theorem 1.1]). If $B(X)$ is the algebra of bounded 
linear operators on a Banach space $X$, then the analyticity of $F: \Omega \rightarrow B(X)$ can be established by verifying that the function $\phi(F(z) x)$ is scalar-valued analytic for any $\phi \in X^{*}$ and $x \in X$ (see [18, p. 267, Theorem 1.2]). Let $w(\zeta)=(1-|\zeta|)^{\beta}$ and $C_{0, w}(\mathbb{D})$ be the Banach space of continuous functions $f: \mathbb{D} \rightarrow \mathbb{C}$ such that $f w$ is bounded and vanishes on $\mathbb{T}$, with the norm of $f$ in $C_{0, w}(\mathbb{D})$ given by $\|w f\|_{\infty}$. For fixed $f \in C_{0}(\mathbb{D})$, the mapping $A: S \rightarrow C_{0, w}(\mathbb{D})$ given by

$$
z \mapsto f(\cdot)(1-|\cdot|)^{-z}
$$

is analytic, because $f(\zeta)(1-|\zeta|)^{\beta-z}$ is bounded in a neighbourhood of any fixed $z \in S$ (we use that $\operatorname{Re} z<\beta$ here), so we easily see that

$$
S \ni z \mapsto \int_{\mathbb{D}} f(\zeta)(1-|\zeta|)^{\beta-z} d \mu(\zeta) \in \mathbb{C}
$$

is scalar-valued analytic for each finite Borel measure $\mu$ on $\mathbb{D}$. Consequently $A^{\prime}: S \rightarrow C_{0}(\mathbb{D})$ given by

$$
z \mapsto \exp \left(\frac{-\epsilon}{1-|\cdot|}\right)\left(R h_{z}\right)(\cdot)
$$

is analytic, because it is equal to $A$ composed with bounded linear maps. Let $M(z): C_{0}(\mathbb{D}) \rightarrow C_{0}(\mathbb{D})$ be the bounded linear operator of multiplication by $(1-|\cdot|)^{z}$. Then $M: S \rightarrow B\left(C_{0}(\mathbb{D})\right)$ is analytic, because

$$
S \ni z \rightarrow \int_{\mathbb{D}}(1-|\zeta|)^{z} g(\zeta) d \mu(\zeta)
$$

is clearly analytic for any finite Borel measure $\mu$ on $\mathbb{D}$ and every $g \in C_{0}(\mathbb{D})$. We conclude that $G_{\epsilon}(z)=M(z) A^{\prime}(z)$ is analytic in $S$.

\section{Boundedness and Compactness}

A consequence of the relation $\left(A_{0}^{-\alpha}\right)^{* *}=A^{-\alpha}$ is that any bounded linear operator $T$ defined on $A_{0}^{-\alpha}$ has an extension to $A^{-\alpha}$ which coincides with the double Banach space adjoint $T^{* *}: A^{-\alpha} \rightarrow A^{-\alpha}$. The operator $T^{* *}$ preserves weak-* convergence of sequences in $A^{-\alpha}$, so that if $f \in A^{-\alpha}$, then

$$
T^{* *} f(z)=\lim _{r \rightarrow 1^{-}} T^{* *} f_{r}(z)=\lim _{r \rightarrow 1^{-}} T f_{r}(z) .
$$

Applying this to the case of $T=T_{g}$ acting on $A_{0}^{-\alpha}$, we see that its double adjoint coincides with $T_{g}$ acting on $A^{-\alpha}$. It follows that if $T_{g}$ is bounded or compact on $A_{0}^{-\alpha}$, then it is also bounded or compact on $A^{-\alpha}$. The converse is a part of the following result.

Proposition 3.1. Let $g: \mathbb{D} \rightarrow \mathbb{C}$ be an analytic function.

(i) The operator $T_{g}$ is bounded on $A^{-\alpha}$ or $A_{0}^{-\alpha}$ if and only if $g \in \mathcal{B}$.

(ii) The operator $T_{g}$ is compact on $A^{-\alpha}$ or $A_{0}^{-\alpha}$ if and only $g \in \mathcal{B}_{0}$.

(iii) The operator norm of $T_{g}$ satisfies

$$
\left\|T_{g}\right\|_{A^{-\alpha}} \leq \frac{\|g\|_{\mathcal{B}}}{\alpha}
$$


Proof. Part (i) and (ii) in the case of $A^{-\alpha}$, as well as part (iii), have already been established in [17], thus we only need to verify (i) and (ii) in the case of $A_{0}^{-\alpha}$. The remark preceeding the proposition implies that if $T_{g}$ is bounded on $A_{0}^{-\alpha}$, then it is bounded on $A^{-\alpha}$, and hence $g \in \mathcal{B}$. Conversely if $g \in \mathcal{B}$, then $T_{g}$ is bounded on $A^{-\alpha}$, and it is easy to verify that it maps the polynomials into $A_{0}^{-\alpha}$. Indeed, if $f \in A_{0}^{-\alpha}$ is a polynomial, then

$$
\begin{aligned}
(1 & -|z|)^{\alpha}\left|\int_{0}^{z} g^{\prime}(\zeta) f(\zeta) d \zeta\right| \\
& \lesssim(1-|z|)^{\alpha}\|f\|_{\infty}\|g\|_{\mathcal{B}} \int_{0}^{1} \frac{|z|}{(1-t|z|)} d t \\
& \leq C(1-|z|)^{\alpha} \log \left(\frac{1}{1-|z|}\right) .
\end{aligned}
$$

The last quantity tends to zero as $|z| \rightarrow 1$, so that $T_{g} f \in A_{0}^{-\alpha}$. Since the polynomials are dense in $A_{0}^{-\alpha}$ and $T_{g}$ is bounded on $A^{-\alpha}$, we obtain $T_{g} A_{0}^{-\alpha} \subset$ $A_{0}^{-\alpha}$. This completes the proof of $(i)$. The same reasoning, with obvious modificiations, leads to a proof of (ii).

We note the following useful consequence of Proposition 3.1 and the discussion preceeding it.

Corollary 3.2. The spectra $\sigma\left(T_{g} \mid A_{0}^{-\alpha}\right)$ and $\sigma\left(T_{g} \mid A^{-\alpha}\right)$ coincide.

Proof. We have verified that $T_{g}$ acts boundedly on $A_{0}^{-\alpha}$ if and only if it acts boundedly on $A^{-\alpha}$. For any bounded linear operator $T$ on a Banach space we have that $\sigma(T)=\sigma\left(T^{*}\right)$. Then the claim follows from this, since the double adjoint of $T_{g}$ acting on $A_{0}^{-\alpha}$ equals $T_{g}$ acting on $A^{-\alpha}$.

\section{Projections on $L_{w}^{\infty}$}

The purpose of this section is to establish some equivalent conditions of boundedness of the standard weighted Bergman projections on the spaces $L_{w}^{\infty}$ which were defined in the introduction. We recall that the definition of the standard weighted Bergman projection $P_{\delta}$, for $\delta>-1$, is

$$
P_{\delta} f(z)=(\delta+1) \int_{\mathbb{D}} f(\zeta) \frac{\left(1-|\zeta|^{2}\right)^{\delta}}{(1-z \bar{\zeta})^{2+\delta}} d A(\zeta)
$$

where $f$ can be any measurable function for which the above integral makes sense. We also introduce the helpful sublinear operator $\tilde{P}$ :

$$
\tilde{P}_{\delta} f(z)=(\delta+1) \int_{\mathbb{D}}|f(\zeta)| \frac{\left(1-|\zeta|^{2}\right)^{\delta}}{|1-z \bar{\zeta}|^{2+\delta}} d A(\zeta) .
$$

Our basic proposition on boundedness of these operators is the following.

Theorem 4.1. Let $w: \mathbb{D} \rightarrow(0, \infty)$ be a weight and $\delta>-1$. The following are equivalent:

(i) The operator $P_{\delta}$ is bounded on $L_{w}^{\infty}$.

(ii) The operator $\tilde{P}_{\delta}$ is bounded on $L_{w}^{\infty}$. 
(iii) The weight $w$ satisfies

$$
\sup _{z \in \mathbb{D}} w(z) \int_{\mathbb{D}} \frac{1}{w(\zeta)} \frac{\left(1-|\zeta|^{2}\right)^{\delta}}{|1-z \bar{\zeta}|^{\delta+2}} d A(\zeta)<\infty .
$$

Proof. $(i) \Rightarrow($ ii $)$ : Fix any $f \in L_{w}^{\infty}$. For any $z \in \mathbb{D}$ there exists a measurable function $u_{z, f}: \mathbb{D} \rightarrow \mathbb{C}$ with $\left|u_{z, f}(\zeta)\right|=1$ for $\zeta \in \mathbb{D}$ such that

$$
\frac{|f(\zeta)|}{|1-z \bar{\zeta}|^{2+\delta}}=\frac{f(\zeta) u_{z, f}(\zeta)}{(1-z \bar{\zeta})^{2+\delta}}
$$

Let $C>0$ be the operator norm of $P_{\delta}$ on $L_{\infty}^{w}$. Then we have

$$
w(z)\left|\tilde{P}_{\delta} f(z)\right|=w(z)\left|P_{\delta} f u_{z, f}(z)\right| \leq C\left\|w f u_{z, f}\right\|_{\infty}=C\|w f\|_{\infty}
$$

which shows that $\tilde{P}_{\delta}$ is bounded on $L_{w}^{\infty}$.

$($ ii $) \Rightarrow($ iii $)$ : The function $w^{-1}$ is in $L_{w}^{\infty}$, and so the boundedness of $\tilde{P}_{\delta}$ implies that

$$
\sup _{z \in \mathbb{D}} w(z) \tilde{P}_{\delta} w^{-1}(z)<\infty,
$$

which is precisely the condition $($ iii $)$.

$($ iii $) \Rightarrow(i)$ : For any $f \in L_{w}^{\infty}$ we have

$$
w(z)\left|P_{\delta} f(z)\right| \leq\|w f\|_{\infty} w(z) \int_{\mathbb{D}} \frac{1}{w(\zeta)} \frac{\left(1-|\zeta|^{2}\right)^{\delta}}{|1-z \bar{\zeta}|^{2+\delta}} d A(\zeta)
$$

Hence if $($ iii $)$ holds, then $P_{\delta}$ is bounded on $L_{w}^{\infty}$.

With the investigation of spectra of $T_{g}$ and weights of the form

$$
w(z)=\left|e^{\frac{g(z)}{\lambda}}\right|\left(1-|z|^{2}\right)^{\alpha}
$$

in mind, we will restrict our further investigation to a class of weights which share some crucial properties with the above. Therefore, we will additionally assume that our weights are bounded, differentiable and satisfy

$$
(1-|z|)|\nabla w(z)| \leq k_{w} w(z)
$$

for some constant $k_{w}>0$. Here $\nabla w$ denotes the gradient of the function $w$. An easily verifiable property of such weights is that they are approximately constant on discs of the form

$$
D_{z}=\{\zeta \in \mathbb{D}:|\zeta-z|<(1-|z|) / 2\}
$$

i.e., there exists a constant $C>0$, independent of $z$, such that $w(\zeta) / w(s) \leq C$ whenever $\zeta, s \in D_{z}$. We also have the growth estimates

$$
w(0)(1-|z|)^{k_{w}} \lesssim w(z) \lesssim w(0)(1-|z|)^{-k_{w}} .
$$

We refer to [2] for proofs of the above claims, where weights satisfying (5) have been studied in the context of $T_{g}$ acting on the weighted Bergman spaces.

For $w$ satisfying the estimate (5) we will now extend Theorem 4.1 to include a fourth equivalent condition, one which will be important in the next section. 
Proposition 4.2. Let $w: \mathbb{D} \rightarrow(0, \infty)$ be a bounded and differentiable weight which satisfies (5). If for some $\delta>-1$ the projection $P_{\delta}$ is a bounded operator on $L_{w}^{\infty}$, then for each integer $n \geq 1$ we have that

$$
\sup _{z \in \mathbb{D}} w(z)|f(z)| \sim \sup _{z \in \mathbb{D}}\left(1-|z|^{2}\right)^{n} w(z)\left|f^{(n)}(z)\right|+\sum_{k=0}^{n-1}\left|f^{(k)}(0)\right|
$$

for all $f \in H_{w}^{\infty}$, i.e., for each integer $n \geq 1$ the right-hand side above defines an equivalent norm on $H_{w}^{\infty}$.

Proof. We start by establishing the proposition in the special case that $n=1$. One of the norm inequalities holds without any assumption on the boundedness of $P_{\delta}$ on $L_{w}^{\infty}$, we need only the fact that $w$ satisfies (5). Indeed, the value $w(z)$ is comparable to values of $w$ on the circle

$$
C_{z}=\{\zeta \in \mathbb{D}:|\zeta-z|=(1-|z|) / 2\},
$$

and so from Cauchy's integral formula we have that

$$
\left|f^{\prime}(z)\right| \lesssim \frac{\sup _{\zeta \in C_{z}}|f(\zeta)|}{1-|z|} \sim \frac{\sup _{\zeta \in C_{z}}|f(\zeta) w(\zeta)|}{(1-|z|) w(z)},
$$

which clearly implies that

$$
(1-|z|) w(z)\left|f^{\prime}(z)\right| \lesssim\|w f\|_{\infty} .
$$

We proceed to establish the reverse inequality. We claim that boundedness of $P_{\delta}$ on $L_{w}^{\infty}$ implies that the operator $P_{\delta}^{1}$ given by

$$
P_{\delta}^{1} h(z)=\int_{\mathbb{D}} h(\zeta) \frac{\left(1-|\zeta|^{2}\right)^{\delta}}{\bar{\zeta}(1-z \bar{\zeta})^{2+\delta}} d A(\zeta)
$$

is bounded on $L_{w}^{\infty}$. To see this, we recall that by Theorem 4.1 the operator $\tilde{P}_{\delta}$ is also bounded on $L_{w}^{\infty}$, and we estimate

$$
\begin{aligned}
w(z)\left|P_{\delta}^{1} h(z)\right| & \leq w(z)\left(\int_{|\zeta|>1 / 2}+\int_{|\zeta|<1 / 2}|h(\zeta)| \frac{\left(1-|\zeta|^{2}\right)^{\delta}}{|\zeta||1-z \bar{\zeta}|^{2+\delta}} d A(\zeta)\right) \\
& \lesssim\|h w\|_{\infty}+\int_{|\zeta|<1 / 2} \frac{|h(\zeta)|}{|\zeta|} d A(\zeta) \\
& \lesssim\|h w\|_{\infty}+\|h w\|_{\infty} \int_{|\zeta|<1 / 2} \frac{1}{|\zeta|} d A(\zeta) \lesssim\|h w\|_{\infty}
\end{aligned}
$$

where in the next-to-last step we used that $w$ is strictly positive, and hence bounded from below for $|\zeta|<1 / 2$. This shows boundedness of $P_{\delta}^{1}$ on $L_{w}^{\infty}$. Now fix a function $f \in H_{w}^{\infty}$ and let

$$
\begin{aligned}
& h(\zeta)=f^{\prime}(\zeta)\left(1-|\zeta|^{2}\right) \in L_{w}^{\infty}, \\
& g(z)=\int_{\mathbb{D}} f^{\prime}(\zeta) \frac{\left(1-|\zeta|^{2}\right)^{\delta+1}}{\bar{\zeta}(1-z \bar{\zeta})^{2+\delta}} d A(\zeta)=P_{\delta}^{1} h(z) .
\end{aligned}
$$

Differentiating $g$ we obtain

$$
g^{\prime}(z)=(\delta+2) \int_{\mathbb{D}} f^{\prime}(\zeta) \frac{\left(1-|\zeta|^{2}\right)^{\delta+1}}{(1-z \bar{\zeta})^{3+\delta}} d A(\zeta)=f^{\prime}(z)
$$


where the last equality is the reproducing property of $P_{\delta+1}$. Hence

$$
f(z)-f(0)=g(z),
$$

because by direct calculation we see that $g(0)=0$. Then the boundedness of $P_{\delta}^{1}$ gives

$$
\left\|w P_{\delta}^{1} h\right\|_{\infty}=\|w(f-f(0))\|_{\infty} \leq\|w h\|_{\infty}
$$

which obviously implies

$$
\sup _{z \in \mathbb{D}} w(z)|f(z)| \lesssim \sup _{z \in \mathbb{D}}\left(1-|z|^{2}\right) w(z)\left|f^{\prime}(z)\right|+|f(0)| .
$$

The proof of the case $n=1$ is complete.

To prove the theorem for $n>1$, note that if $P_{\delta}$ is bounded on $L_{w}^{\infty}$, then $P_{\delta+1}$ is bounded on $L_{\tilde{w}}^{\infty}$, where $\tilde{w}(\zeta)=\left(1-|\zeta|^{2}\right) w(\zeta)$. This follows from Theorem 4.1, because we have

$$
\begin{aligned}
\tilde{w}(z) \int_{\mathbb{D}} \frac{1}{\tilde{w}(\zeta)} \frac{\left(1-|\zeta|^{2}\right)^{\delta+1}}{|1-z \bar{\zeta}|^{\delta+3}} d A(\zeta) & =w(z)\left(1-|z|^{2}\right) \int_{\mathbb{D}} \frac{1}{w(\zeta)} \frac{\left(1-|\zeta|^{2}\right)^{\delta}}{|1-z \bar{\zeta}|^{\delta+3}} d A(\zeta) \\
& \lesssim w(z) \int_{\mathbb{D}} \frac{1}{w(\zeta)} \frac{\left(1-|\zeta|^{2}\right)^{\delta}}{|1-z \bar{\zeta}|^{\delta+2}} d A(\zeta) .
\end{aligned}
$$

The case $n>1$ of the proposition now follows readily by induction.

We proceed to prove the converse of Proposition 4.2. For this, we will need the following very useful result from [2, Lemma 3.2].

Lemma 4.3. Let $w: \mathbb{D} \rightarrow(0, \infty)$ be a differentiable weight which satisfies $(5)$. If $\alpha+1>k_{w}$ and $\beta>\alpha+2+k_{w}$, then

$$
\int_{\mathbb{D}} w(\zeta) \frac{\left(1-|\zeta|^{2}\right)^{\alpha}}{|1-z \bar{\zeta}|^{\beta}} d A(\zeta) \lesssim \frac{w(z)}{(1-|z|)^{\beta-\alpha-2}} .
$$

Proposition 4.4. Let $w: \mathbb{D} \rightarrow(0, \infty)$ be a bounded and differentiable weight which satisfies (5). If for each $n \geq 1$

$$
\sup _{z \in \mathbb{D}}\left(1-|z|^{2}\right)^{n} w(z)\left|f^{(n)}(z)\right|+\sum_{k=1}^{n-1}\left|f^{(k)}(0)\right|
$$

defines an equivalent norm on $H_{w}^{\infty}$, then there exists $a \delta>-1$ such that $P_{\delta}$ is bounded on $L_{w}^{\infty}$.

Proof. Since $w$ satisfies (6), we see that for sufficiently large $\delta$ the integral defining $P_{\delta} f$ converges for every $f \in L_{w}^{\infty}$. We will show that $\delta$ can be chosen so that the operator $P_{\delta}$ is bounded on $L_{w}^{\infty}$. This will follow from the assumption if we show that for some $n \geq 0$ we have

$$
w(z)\left(1-|z|^{2}\right)^{n}\left|\int_{\mathbb{D}} f(\zeta) \frac{\left(1-|\zeta|^{2}\right)^{\delta}}{(1-z \bar{\zeta})^{\delta+2+n}} d A(\zeta)\right| \leq C\|f w\|_{\infty} .
$$

We have the obvious estimate

$$
\left|\int_{\mathbb{D}} f(\zeta) \frac{\left(1-|\zeta|^{2}\right)^{\delta}}{(1-z \bar{\zeta})^{\delta+2+n}} d A(\zeta)\right| \leq\|f w\|_{\infty} \int_{\mathbb{D}} \frac{1}{w(\zeta)} \frac{\left(1-|\zeta|^{2}\right)^{\delta}}{|1-z \bar{\zeta}|^{\delta+2+n}} d A(\zeta) .
$$


An easy computation shows that the weight $\tilde{w}=1 / w$ also satisfies (5), so if $n$ and $\delta$ are sufficiently large, then by Lemma 4.3 we obtain

$$
\int_{\mathbb{D}} \frac{1}{w(\zeta)} \frac{\left(1-|\zeta|^{2}\right)^{\delta}}{|1-z \bar{\zeta}|^{\delta+2+n}} d A(\zeta) \leq C \frac{1}{w(z)(1-|z|)^{n}} .
$$

Proposition 4.2 and Proposition 4.4 now imply the following version of Theorem 4.1.

Theorem 4.5. Let $w: \mathbb{D} \rightarrow(0, \infty)$ be a bounded and differentiable weight which satisfies (5). The following are equivalent:

(i) The operator $P_{\delta}$ is bounded on $L_{w}^{\infty}$ for some $\delta>-1$.

(ii) The operator $\tilde{P}_{\delta}$ is bounded on $L_{w}^{\infty}$ for some $\delta>-1$

(iii) There exists $\delta>-1$ such that

$$
\sup _{z \in \mathbb{D}} w(z) \int_{\mathbb{D}} \frac{1}{w(\zeta)} \frac{\left(1-|\zeta|^{2}\right)^{\delta}}{|1-z \bar{\zeta}|^{\delta+2}} d A(\zeta)<\infty .
$$

(iv) For each $n \geq 1$,

$$
\sup _{z \in \mathbb{D}}\left(1-|z|^{2}\right)^{n} w(z)\left|f^{(n)}(z)\right|+\sum_{k=0}^{n-1}\left|f^{(k)}(0)\right|
$$

defines an equivalent norm on $H_{w}^{\infty}$.

\section{Spectrum}

As mentioned in the introduction, for fixed analytic functions $f, h: \mathbb{D} \rightarrow \mathbb{C}$, the equation

$$
h-\frac{1}{\lambda} T_{g} h=f
$$

has a unique solution $R_{\lambda, g} f$ which is given by

$$
R_{\lambda, g} f(z)=f(0) e^{\frac{g(z)}{\lambda}}+e^{\frac{g(z)}{\lambda}} \int_{0}^{z} e^{-\frac{g(\zeta)}{\lambda}} f^{\prime}(\zeta) d \zeta .
$$

It is easy to see that $T_{g}$ is injective if $g \neq 0$, and therefore non-zero $\lambda$ is in the resolvent set of $T_{g}$ acting on any Banach space $X$ of analytic functions if and only if the operator $R_{\lambda, g}$ is bounded on $X$. If this is the case, then $R_{\lambda, g}$ is automatically invertible on $X$.

\subsection{Spectrum}

We will now apply the results of Sect. 4 to prove Theorem 5.3, which provides a characterization of the spectrum of $T_{g}$ acting on $A^{-\alpha}$.

Proposition 5.1. The operator $R_{\lambda, g}$ is bounded on $A^{-\alpha}$ if and only if

$$
e^{g / \lambda} \in A^{-\alpha}
$$

and the weight

$$
w(z)=\left|e^{\frac{g(z)}{\lambda}}\right|\left(1-|z|^{2}\right)^{\alpha}
$$


satisfies

$$
\sup _{z \in \mathbb{D}} w(z)|f(z)| \sim \sup _{z \in \mathbb{D}}\left(1-|z|^{2}\right) w(z)\left|f^{\prime}(z)\right|+|f(0)|
$$

for every $f \in H_{w}^{\infty}(\mathbb{D})$.

Proof. Assume first that $R_{\lambda, g}$ is bounded on $A^{-\alpha}$. Then $e^{g / \lambda}=R_{\lambda, g} 1 \in A^{-\alpha}$, so that (7) holds. Moreover, note that $f \in H_{w}^{\infty}$ if and only if $e^{g / \lambda} f \in A^{-\alpha}$, and that if $f(0)=0$, then

$$
e^{g / \lambda} f=R_{\lambda, g} \int e^{g / \lambda} f^{\prime} .
$$

Hence by the invertibility of $R_{\lambda, g}$ we have that $\int e^{g / \lambda} f^{\prime} \in A^{-\alpha}$ and

$$
\left\|e^{g / \lambda} f\right\|_{-\alpha} \sim\left\|e^{g / \lambda} f^{\prime}\right\|_{-(\alpha+1)}
$$

which is clearly equivalent to (8). Conversely, assume that (7) and (8) hold. Then $R_{\lambda, g}$ maps constants into $A^{-\alpha}$ because $R_{\lambda, g} 1=e^{g / \lambda}$, while for any $f \in A^{-\alpha}$ with $f(0)=0$ we have

$$
\begin{aligned}
\left\|R_{\lambda, g} f\right\|_{-\alpha}= & \sup _{z \in \mathbb{D}} w(z)\left|\int_{0}^{z} e^{-g(\zeta) / \lambda} f^{\prime}(\zeta) d \zeta\right| \\
& \sim \sup _{z \in \mathbb{D}}\left(1-|z|^{2}\right)^{\alpha+1}\left|f^{\prime}(z)\right|<\infty .
\end{aligned}
$$

We make a short remark. By Corollary $3.2, R_{\lambda, g}$ is bounded on $A^{-\alpha}$ if and only if it is bounded on $A_{0}^{-\alpha}$. Since $1 \in A_{0}^{-\alpha}$, we see that if $R_{\lambda, g}$ is bounded on $A^{-\alpha}$, then we obtain the stronger statement that $e^{g / \lambda} \in A_{0}^{-\alpha}$.

Proposition 5.2. Assume that $0 \neq \lambda \in \rho\left(T_{g} \mid A^{-\alpha}\right)$ and let

$$
w(z)=\left|e^{\frac{g(z)}{\lambda}}\right|\left(1-|z|^{2}\right)^{\alpha} .
$$

For each $n \geq 1$,

$$
\sup _{z \in \mathbb{D}}\left(1-|z|^{2}\right)^{n} w(z)\left|f^{(n)}(z)\right|+\sum_{k=0}^{n-1}\left|f^{(k)}(0)\right|
$$

defines an equivalent norm on $H_{w}^{\infty}$.

Proof. The assumption that $\lambda \in \rho\left(T_{g} \mid A^{-\alpha}\right)$ mean precisely that $R=R_{\lambda, g}$ is bounded on $A^{-\alpha}$, and by Corollary 3.2 it is also bounded on $A_{0}^{-\alpha}$. For any sufficiently large $\beta>0$ we have by Proposition 3.1 that $|\lambda|>\left\|T_{g}\right\|_{A^{-\beta}}$, and for such $\beta$ the operator $R_{\lambda}, g$ is bounded on $A_{0}^{-\beta}$. Then from Theorem 2.2 and Corollary 3.2 we obtain that $R_{\lambda, g}$ is bounded on $A^{-\beta}$ for all $\beta>\alpha$, and the claim follows from Proposition 5.1 by induction.

We can now prove the main result characterizing the spectrum of $T_{g}$ acting on $A^{-\alpha}$.

Theorem 5.3. Assume that $g \in \mathcal{B}, \lambda \in \mathbb{C} \backslash\{0\}$ and

$$
w(z)=\left|e^{\frac{g(z)}{\lambda}}\right|\left(1-|z|^{2}\right)^{\alpha} .
$$

The following are equivalent: 
(i) $\lambda \in \rho\left(T_{g} \mid A^{-\alpha}\right)$.

(ii) For some $\delta>-1$, the weight $w$ satisfies

$$
\sup _{z \in \mathbb{D}} w(z) \int_{\mathbb{D}} \frac{1}{w(\zeta)} \frac{\left(1-|\zeta|^{2}\right)^{\delta}}{|1-z \bar{\zeta}|^{\delta+2}} d A(\zeta)<\infty .
$$

Proof. The implication $(i) \Rightarrow(i i)$ is covered by Proposition 5.2 together with Theorem 4.5. If $(i i)$ holds, then $w(z)$ is certainly bounded, and this together with Theorem 4.5 implies that the two conditions of Proposition 5.1 hold, so that $(i i)$ implies $(i)$.

\subsection{Applications}

As a first application of Theorem 5.3 we establish the spectral stability property of the operator $T_{g}$ that was mentioned in the introduction. Next we obtain the spectrum of $T_{g}$ in the case $g$ is the anti-derivative of a rational function. The two results are then combined in Theorem 5.6 to obtain the spectrum of $T_{g}$ whenever $g=r+h+b$, where $r^{\prime}$ is rational, $h \in H^{\infty}$ and $b \in \mathcal{B}_{0}$.

Theorem 5.4. Let $g, h \in \mathcal{B}$ and assume that $\sigma\left(T_{h} \mid A^{-\alpha}\right)=\{0\}$. Then

$$
\sigma\left(T_{g+h} \mid A^{-\alpha}\right)=\sigma\left(T_{g} \mid A^{-\alpha}\right) .
$$

Proof. It will be sufficient to show that $\rho\left(T_{g} \mid A^{-\alpha}\right) \subseteq \rho\left(T_{g+h} \mid A^{-\alpha}\right)$, since the other inclusion follows by replacing $g$ with $g+h$ and $h$ with $-h$. Fix $\lambda \in \rho\left(T_{g} \mid A^{-\alpha}\right)$. By Theorem 5.3 we must verify that there exists a $\delta>-1$ such that

$$
\sup _{z \in \mathbb{D}}\left(1-|z|^{2}\right)^{\alpha}\left|e^{g(z) / \lambda}\right|\left|e^{h(z) / \lambda}\right| \int_{\mathbb{D}}\left|e^{-g(\zeta) / \lambda}\right|\left|e^{-h(\zeta) / \lambda}\right| \frac{\left(1-|\zeta|^{2}\right)^{\delta-\alpha}}{|1-z \bar{\zeta}|^{\delta+2}} d A(\zeta)<\infty .
$$

Take $p, q>1$ with $1 / p+1 / q=1$ and $p$ close enough to 1 so that $\tilde{\lambda}=\lambda / p \in$ $\rho\left(T_{g} \mid A^{-\alpha}\right)$. Let $\hat{\lambda}=\lambda / q$. Use Hölder's inequality to obtain

$$
\begin{aligned}
\int_{\mathbb{D}}\left|e^{-g(\zeta) / \lambda}\right|\left|e^{-h(\zeta) / \lambda}\right| \frac{\left(1-|\zeta|^{2}\right)^{\delta-\alpha}}{|1-z \bar{\zeta}|^{\delta+2}} d A(\zeta) \\
\leq\left(\int_{\mathbb{D}}\left|e^{-g(\zeta) / \tilde{\lambda}}\right| \frac{\left(1-|\zeta|^{2}\right)^{\delta-\alpha}}{|1-z \bar{\zeta}|^{\delta+2}} d A(\zeta)\right)^{1 / p} \\
\quad \times\left(\int_{\mathbb{D}}\left|e^{-h(\zeta) / \hat{\lambda}}\right| \frac{\left(1-|\zeta|^{2}\right)^{\delta-\alpha}}{|1-z \bar{\zeta}|^{\delta+2}} d A(\zeta)\right)^{1 / q}
\end{aligned}
$$

Now use the assumption that $\hat{\lambda} \in \rho\left(T_{h} \mid A^{-\alpha}\right)=\mathbb{C} \backslash\{0\}$ and Theorem 5.3 to see that $\delta>-1$ can be chosen big enough so that we simultaneously have

$$
\sup _{z \in \mathbb{D}}\left(1-|z|^{2}\right)^{\alpha}\left|e^{g(z) / \tilde{\lambda}}\right| \int_{\mathbb{D}}\left|e^{-g(\zeta) / \tilde{\lambda}}\right| \frac{\left(1-|\zeta|^{2}\right)^{\delta-\alpha}}{|1-z \bar{\zeta}|^{\delta+2}} d A(\zeta)=C_{1}<\infty
$$

and

$$
\sup _{z \in \mathbb{D}}\left(1-|z|^{2}\right)^{\alpha}\left|e^{h(z) / \hat{\lambda}}\right| \int_{\mathbb{D}}\left|e^{-h(\zeta) / \hat{\lambda}}\right| \frac{\left(1-|\zeta|^{2}\right)^{\delta-\alpha}}{|1-z \bar{\zeta}|^{\delta+2}} d A(\zeta)=C_{2}<\infty .
$$


We get that

$$
\begin{aligned}
(1- & \left.|z|^{2}\right)^{\alpha}\left|e^{g(z) / \lambda}\right|\left|e^{h(z) / \lambda}\right| \int_{\mathbb{D}}\left|e^{-g(\zeta) / \lambda}\right|\left|e^{-h(\zeta) / \lambda}\right| \frac{\left(1-|\zeta|^{2}\right)^{\delta-\alpha}}{|1-z \bar{\zeta}|^{\delta+2}} d A(\zeta) \\
\leq & \left(1-|z|^{2}\right)^{\alpha}\left|e^{g(z) / \lambda}\right|\left|e^{h(z) / \lambda}\right|\left(\int_{\mathbb{D}}\left|e^{-g(\zeta) / \tilde{\lambda}}\right| \frac{\left(1-|\zeta|^{2}\right)^{\delta-\alpha}}{|1-z \bar{\zeta}|^{\delta+2}} d A(\zeta)\right)^{1 / p} \\
& \times\left(\int_{\mathbb{D}}\left|e^{-h(\zeta) / \hat{\lambda}}\right| \frac{\left(1-|\zeta|^{2}\right)^{\delta-\alpha}}{|1-z \bar{\zeta}|^{\delta+2}} d A(\zeta)\right)^{1 / q} \\
= & \left(\left(1-|z|^{2}\right)^{\alpha}\left|e^{g(z) / \tilde{\lambda}}\right| \int_{\mathbb{D}}\left|e^{-g(\zeta) / \tilde{\lambda}}\right| \frac{\left(1-|\zeta|^{2}\right)^{\delta-\alpha}}{|1-z \bar{\zeta}|^{\delta+2}} d A(\zeta)\right)^{1 / p} \\
& \times\left(\left(1-|z|^{2}\right)^{\alpha}\left|e^{h(z) / \hat{\lambda}}\right| \int_{\mathbb{D}}\left|e^{-h(\zeta) / \hat{\lambda}}\right| \frac{\left(1-|\zeta|^{2}\right)^{\delta-\alpha}}{|1-z \bar{\zeta}|^{\delta+2}} d A(\zeta)\right)^{1 / q} \\
\leq & C_{1}^{1 / p} C_{2}^{1 / q} .
\end{aligned}
$$

Corollary 5.5. Let $g, h \in \mathcal{B}$ and assume that $h \in H^{\infty}$ or $h \in \mathcal{B}_{0}$. Then

$$
\sigma\left(T_{g+h} \mid A^{-\alpha}\right)=\sigma\left(T_{g} \mid A^{-\alpha}\right) .
$$

Proof. By Proposition 3.1 if $h \in \mathcal{B}_{0}$, then $T_{h}$ is compact and hence $\sigma\left(T_{h} \mid A^{-\alpha}\right)$ $=\{0\}$ since $T_{g}$ has no eigenvalues. On the other hand if $h \in H^{\infty}$ then for all $\lambda \neq 0$ the function $e^{h / \lambda}$ is bounded from above and below in $\mathbb{D}$. It then follows from Proposition 2.1 that condition $(i i)$ of Theorem 5.3 is satisfied, so that again $\sigma\left(T_{h} \mid A^{-\alpha}\right)=\{0\}$. The claim then follows from Theorem 5.4.

Let $\omega_{0}, \ldots, \omega_{n}$ be distinct points on the circle $\mathbb{T}$. For non-zero complex numbers $c_{0}, \ldots, c_{n}$ let

$$
g(z)=\sum_{k=0}^{n} c_{k} \log \left(\frac{1}{1-\overline{\omega_{k}} z}\right) \in \mathcal{B} .
$$

The spectrum of $T_{g}$ in this case turns out to be

$$
\sigma\left(T_{g} \mid A^{-\alpha}\right)=\bigcup_{k=1}^{n}\left\{\lambda \in \mathbb{C}: \operatorname{Re}\left(c_{k} / \lambda\right) \geq \alpha\right\} .
$$

This result can be predicted from results in [4], where it is shown that the equality above holds with $T_{g}$ replaced by the operator $f \mapsto \frac{1}{z} T_{g} f$. We shall therefore not carry out the entire argument, but merely indicate how condition ( $i i)$ of Theorem 5.3 can be used to establish (9). Let $s_{k}=\operatorname{Re}\left(c_{k} / \lambda\right)$. Since for each $k$ the function $\log \left(\frac{1}{1-\overline{\omega_{k}} z}\right)$ has bounded imaginary part, it follows easily that

$$
\left|e^{g(z) / \lambda}\right| \sim \prod_{k=0}^{n}\left|1-\overline{\omega_{k}} z\right|^{-s_{k}}
$$


If for some $k$ we have that $s_{k}>\alpha$, then by the above $e^{g / \lambda} \notin A^{-\alpha}$, which by Proposition 5.1 implies that

$$
\bigcup_{k=1}^{n}\left\{\lambda \in \mathbb{C}: \operatorname{Re}\left(c_{k} / \lambda\right) \geq \alpha\right\} \subseteq \sigma\left(T_{g}: A^{-\alpha}\right) .
$$

Conversely, if $s_{k}<\alpha$ for $k=0, \ldots, n$, then to show that $\lambda \in \rho\left(T_{g} \mid A^{-\alpha}\right)$ it will suffice by (10) and (ii) of Theorem 5.3 to show that

$$
\sup _{z \in \mathbb{D}}\left(1-|z|^{2}\right)^{\alpha} \prod_{k=0}^{n}\left|1-\overline{\omega_{k}} z\right|^{-s_{k}} \int_{\mathbb{D}} \prod_{k=0}^{n}\left|1-\overline{\omega_{k}} \zeta\right|^{s_{k}} \frac{\left(1-|\zeta|^{2}\right)^{\delta-\alpha}}{|1-z \bar{\zeta}|^{\delta+2}} d A(\zeta)<\infty
$$

holds for some $\delta>-1$. This follows from Proposition 2.1 together with a straightforward and elementary computation involving isolation of the possible poles of the functions $\left|1-\bar{\omega}_{k} \zeta\right|^{s_{k}}$.

Theorem 5.6. Let $h \in H^{\infty}, b \in \mathcal{B}_{0}$ and

$$
r(z)=\sum_{k=0}^{n} c_{k} \log \left(\frac{1}{1-\overline{\omega_{k}} z}\right) .
$$

If $g=r+h+b$, then

$$
\begin{aligned}
\sigma\left(T_{g} \mid A^{-\alpha}\right) & =\bigcup_{k=1}^{n}\left\{\lambda \in \mathbb{C}: \operatorname{Re}\left(c_{k} / \lambda\right) \geq \alpha\right\} \\
& =\{0\} \cup \overline{\left\{\lambda \in \mathbb{C} \backslash\{0\}: e^{g / \lambda} \notin A^{-\alpha}\right\}} .
\end{aligned}
$$

Proof. The first of the two set equalities in the statement is immediate from the discussion preceeding the theorem and Corollary 5.5. To establish the second equality it will be sufficient to show that $e^{g / \lambda} \notin A^{-\alpha}$ whenever $\lambda$ is in the interior of one of the closed disks whose union is $\sigma\left(T_{g} \mid A^{-\alpha}\right)$. For any such $\lambda$ there exists an $\epsilon>0$ such that $e^{r / \lambda} \notin A^{-\alpha-\epsilon}$. Since $h \in H^{\infty}$ and $b \in \mathcal{B}_{0}$, we obtain easily that

$$
e^{(h(z)+b(z)) / \lambda} \gtrsim\left(1-|z|^{2}\right)^{\epsilon} .
$$

Then

$$
\sup _{z \in \mathbb{D}}\left(1-|z|^{2}\right)^{\alpha}\left|e^{g(z) / \lambda}\right| \gtrsim \sup _{z \in \mathbb{D}}\left(1-|z|^{2}\right)^{\alpha+\epsilon}\left|e^{r(z) / \lambda}\right|=\infty,
$$

So $e^{g / \lambda} \notin A^{-\alpha}$.

\subsection{An Important Example}

For $p>1$ and $\eta>-1$ the class $B_{p}(\eta)$ consists of weights $w$ on $\mathbb{D}$ for which there exists a constant $C>0$ such that

$$
\begin{aligned}
& \left(\int_{S(\theta, h)} w(\zeta)\left(1-|\zeta|^{2}\right)^{\eta} d A(\zeta)\right)\left(\int_{S(\theta, h)} w(\zeta)^{-q / p}\left(1-|\zeta|^{2}\right)^{\eta} d A(\zeta)\right)^{p / q} \\
& \quad \leq C h^{p(\eta+2)}
\end{aligned}
$$

for any Carleson box $S(\theta, h)$ given by

$$
S(\theta, h)=\left\{z=r e^{i t} \in \mathbb{D}: 1-r<h,|t-\theta|<h\right\} .
$$


The significance of this definition comes from the work of Békollé, who proves that the $B_{p}(\eta)$-condition is related to the boundedness of the standard weighted Bergman projections on weighted Bergman spaces. See [7] for details.

Proposition 5.7. Let $w: \mathbb{D} \rightarrow(0, \infty)$ be a weight. If $w$ satisfies for some $\delta>-1$ the condition (ii) of Theorem 5.3, then

$$
w(z)\left(1-|z|^{2}\right)^{-\eta} \in B_{2}(\eta)
$$

for $\eta=\delta / 2$.

Proof. Let $S=S(\theta, h)$ be a Carleson square of width $h>0$. We have

$$
w(z) \int_{S} \frac{1}{w(\zeta)} \frac{\left(1-|\zeta|^{2}\right)^{\delta}}{|1-z \bar{\zeta}|^{\delta+2}} d A(\zeta) \leq C
$$

for all $z \in \mathbb{D}$, and in particular for $z \in S$. But if $z, \zeta \in S$, then $|1-z \bar{\zeta}| \lesssim h$, and consequently

$$
w(z) \int_{S} \frac{1}{w(\zeta)}\left(1-|\zeta|^{2}\right)^{\delta} d A(\zeta) \lesssim h^{\delta+2}
$$

Now integrate for $z \in S$ to obtain

$$
\int_{S} w(z) d A(z) \int_{S} \frac{1}{w(\zeta)}\left(1-|\zeta|^{2}\right)^{\delta} d A(\zeta) \lesssim h^{\delta+4} .
$$

Setting $\eta=\delta / 2$, the last inequality means precisely that $w(z)\left(1-|z|^{2}\right)^{-\eta} \in$ $B_{2}(\eta)$.

The significance of the above proposition in the study of the spectrum $\sigma\left(T_{g} \mid A^{-\alpha}\right)$ is the following. We have seen in this section that the equality

$$
\sigma\left(T_{g} \mid A^{-\alpha}\right)=\{0\} \cup \overline{\left\{\lambda \in \mathbb{C} \backslash\{0\}: e^{g / \lambda} \notin A^{-\alpha}\right\}}
$$

holds for a large class of symbols $g$. The fact that (11) does not hold for general $g \in \mathcal{B}$ can be seen from Proposition 5.7 and the corresponding result for weighted Bergman spaces $L_{a}^{p, \alpha}$. For $p>0$ and $\alpha>-1$ the space $L_{a}^{p, \alpha}$ consists of functions analytic in $\mathbb{D}$ which satisfy

$$
\|f\|_{p, \alpha}^{p}=\int_{\mathbb{D}}|f(z)|^{p}\left(1-|z|^{2}\right)^{\alpha} d A(z)<\infty .
$$

The operator $T_{g}$ acts boundedly on $L_{a}^{p, \alpha}$ if and only if $g \in \mathcal{B}$, and it is shown in [2] that $\lambda \in \rho\left(T_{g} \mid L_{a}^{p, \alpha}\right)$ if and only if the weight

$$
w(z)=\left|e^{p g(z) / \lambda}\right|\left(1-|z|^{2}\right)^{\alpha}
$$

is integrable (which means precisely that $\left.e^{g / \lambda} \in L_{a}^{p, \alpha}\right)$ and $\tilde{w}(z)=w(z)(1-$ $|z|)^{-\eta}$ satisfies for some $\eta>-1$ and $p_{0}>1$ the $B_{p_{0}}(\eta)$-condition. It is shown in [2] that there exists a function $g \in \mathcal{B}$ such that $e^{g / \lambda}$ belongs to the space $L_{a}^{p, \alpha}$ for all $\lambda \in \mathbb{C} \backslash\{0\}$ and $p>0$, yet the spectrum $\sigma\left(T_{g} \mid L_{a}^{p, \alpha}\right)$ is always larger than $\{0\}$. See $[2$, Section 5] for details of the construction of the function $g$ and its properties. It can be see in [2] that $g$ also satisfies $e^{g / \lambda} \in A_{0}^{-\alpha}$ for all $\lambda \in \mathbb{C} \backslash\{0\}$ and all $\alpha>0$. Let $w(z)=\left|e^{g(z) / \lambda}\right|\left(1-|z|^{2}\right)^{\alpha}$. If $\lambda \in \rho\left(T_{g} \mid A^{-\alpha}\right)$, then condition $(i i)$ of Theorem 5.3 holds for $w$ for sufficiently large $\delta$, and 
consequently by Proposition 5.7 and the results of [2] mentioned above we have that $\lambda \in \rho\left(T_{g} \mid L_{a}^{1, \alpha}\right)$. It follows that

$$
\sigma\left(T_{g} \mid A^{-\alpha}\right) \supseteq \sigma\left(T_{g} \mid L_{a}^{1, \alpha}\right) .
$$

Since $\sigma\left(T_{g} \mid L_{a}^{1, \alpha}\right)$ is bigger than $\{0\}$, thus so is $\sigma\left(T_{g} \mid A^{-\alpha}\right)$.

\section{Acknowledgements}

The author would like to thank his supervisor, Professor Alexandru Aleman, for the suggestion to study this problem and for many helpful discussions.

Open Access. This article is distributed under the terms of the Creative Commons Attribution 4.0 International License (http://creativecommons.org/licenses/by/4. 0/), which permits unrestricted use, distribution, and reproduction in any medium, provided you give appropriate credit to the original author(s) and the source, provide a link to the Creative Commons license, and indicate if changes were made.

\section{References}

[1] Aleman, A.: A class of integral operators on spaces of analytic functions. In: Topics in Complex Analysis and Operator Theory. Univ. Málaga, Málaga, pp. 3-30 (2007)

[2] Aleman, A., Constantin, O.: Spectra of integration operators on weighted Bergman spaces. J. Anal. Math. 109, 199-231 (2009)

[3] Aleman, A., Peláez, J.A.: Spectra of integration operators and weighted square functions. Indiana Univ. Math. J. 61, 775-793 (2012)

[4] Aleman, A., Persson, A.-M.: Resolvent estimates and decomposable extensions of generalized Cesàro operators. J. Funct. Anal. 258, 67-98 (2010)

[5] Aleman, A., Siskakis, A.: An integral operator on $H^{p}$. Complex Var. Theory Appl. 28, 149-158 (1995)

[6] Aleman, A., Siskakis, A.: Integration operators on Bergman spaces. Indiana Univ. Math. J. 46, 337-356 (1997)

[7] Békollé, D.: Inégalité à poids pour le projecteur de Bergman dans la boule unité de $\mathbf{C}^{n}$, Studia Math., 71, pp. 305-323 (1981/82)

[8] Bonet, J.: The spectrum of Volterra operators on weighted spaces of entire functions. Q. J. Math. 66, 799-807 (2015)

[9] Bonet, J., Engliš, M., Taskinen, J.: Weighted $L^{\infty}$-estimates for Bergman projections. Studia Math. 171, 67-92 (2005)

[10] Constantin, O.: A Volterra-type integration operator on Fock spaces. Proc. Am. Math. Soc. 140, 4247-4257 (2012)

[11] Constantin, O., Persson, A.-M.: The spectrum of Volterra-type integration operators on generalized Fock spaces. Bull. Lond. Math. Soc. 47, 958-963 (2015)

[12] Duren, P.L.: Theory of $H^{p}$ Spaces. Pure and Applied Mathematics, vol. 38. Academic Press, New York (1970)

[13] Hedenmalm, H., Korenblum, B., Zhu, K.: Theory of Bergman Spaces. Graduate Texts in Mathematics, vol. 199. Springer, New York (2000) 
[14] Pommerenke, C.: Schlichte Funktionen und analytische Funktionen von beschränkter mittlerer Oszillation. Comment. Math. Helv. 52, 591-602 (1977)

[15] Rubel, L.A., Shields, A.L.: The second duals of certain spaces of analytic functions. J. Austral. Math. Soc. 11, 276-280 (1970)

[16] Siskakis, A.G.: Volterra operators on spaces of analytic functions - a survey. In: Proceedings of the First Advanced Course in Operator Theory and Complex Analysis, Univ. Sevilla Secr. Publ., Seville, pp. 51-68 (2006)

[17] Stević, S.: Boundedness and compactness of an integral operator on a weighted space on the polydisc. Indian J. Pure Appl. Math. 37, 343-355 (2006)

[18] Taylor, A.E., Lay, D.C.: Introduction to Functional Analysis, 2nd edn. Wiley, New York (1980)

[19] Young, S.W.: Spectral properties of generalized Cesàro operators. Integr. Equ. Oper. Theory 50, 129-146 (2004)

Bartosz Malman $(\bowtie)$

Centre for Mathematical Sciences

Lund University

P.O. Box 11822100 Lund

Sweden

e-mail: bartosz.malman@math.lu.se

Received: August 28, 2017.

Revised: March 15, 2018. 